\title{
Vortex Layer on the $\beta$-Plane in the Miles - Ribner Formulation. Pole on the Real Axis
}

\author{
V. G. Gnevyshev ${ }^{1}$, T. V. Belonenko ${ }^{2, 凶}$ \\ ${ }^{1}$ Shirshov Institute of Oceanology, Russian Academy of Sciences, Moscow, Russian Federation \\ ${ }^{2}$ Saint Petersburg State University, St. Petersburg, Russian Federation \\ \tvlisab@yandex.ru
}

\begin{abstract}
Purpose. The problem of a non-zonal vortex layer on the $\beta$-plane in the Miles - Ribner formulation is considered. It is known that in the absence of the $\beta$-effect, the vortex layer has no neutral eigenmodes, and the available two ones (varicose and sinusoidal) are unstable. Initially, generalization of the problem to the $\beta$-plane concerned only the zonal case. The problem for a non-zonal vortex layer is examined for the first time in the paper. It is known that in the WKB approximation for the linear wave disturbances (regardless of whether a zonal or non-zonal background flow is considered), there is an adiabatic invariant in the form of the law of the enstrophy (vorticity) conservation. For the zonal vortex layer, the enstrophy conservation law also holds, and no vorticity exchange occurs between the waves and the flow in the zonal case. The non-zonal vortex layer has qualitatively different features; particularly, it does not retain enstrophy. Thus, as a result, there appears a new class of solutions which can be interpreted as pure radiation of the Rossby waves by a non-zonal flow. Generalizing the vortex layer problem on the $\beta$-plane to the non-zonal case constitutes the basic aim of the present study.

Methods and Results. A new class of linear stationary wave solutions, namely the Rossby waves, is found. It is shown a non-zonal flow can be directed in one way, whereas the stationary wave disturbances can move in the opposite (contrary) direction. The coexistence of such solutions for the shear non-zonal flow and stationary wave disturbances takes place due to the influence of the external force and mathematically comes from a non-self-adjoining character of the linear operator for a non-zonal background flow.

Conclusion. There exists a new class of solutions that can be interpreted as pure radiation of the Rossby waves by a non-zonal flow. There is no such solution for a zonal flow. It is just nonzoning that gives the effect of pure radiation and corresponds to the classical definition of radiation. This approach makes it possible to eliminate inconsistency in terminology, when instabilities are mistakenly called radiation, and radiation - pure radiation.
\end{abstract}

Keywords: Rossby waves, vortex layer, non-zonal flow, Miles - Ribner problem

Acknowledgments: the work was supported by the RFBR grant No. 20-05-00066. The research by V.G. Gnevyshev was also supported by the IO RAS, ST-ASS-0128-2021-0003.

For citation: Gnevyshev, V.G. and Belonenko, T.V., 2021. Vortex Layer on the $\beta$-Plane in the Miles - Ribner Formulation. Pole on the Real Axis. Physical Oceanography, [e-journal] 28(5), pp. 486-498. doi:10.22449/1573-160X-2021-5-486-498

DOI: $10.22449 / 1573-160 \mathrm{X}-2021-5-486-498$

(C) V. G. Gnevyshev, T. V. Belonenko, 2021

(C) Physical Oceanography, 2021

\section{Introduction}

In the analytical description of the interaction of large-scale flows and wave disturbances in a linear formulation, two qualitatively different approaches are used. The first approach is based on the assumption that the wave field of the background flow is described by smooth functions; in this case, either model problems or the WKB approximation are used. In model problems, the physical fields of the background flow (velocity field and stratification) are specified by analytical expressions, and the solution of the problem, as a rule, can be reduced to 
special functions [1-5]. When using the WKB approximation, it is assumed that the background flow changes rather smoothly, the wave has time to rebuild its field to match the current parameters of the background flow, and thus, as a result, we actually obtain a parametric dependence on the background flows [6, 7].

The second approach boils down to rejecting the complete smoothness of the background fields and switching to the models of piecewise continuous profiles of the background flow. The simplest formulation of such problems is a vortex layer problem when the velocity field consists of two different constants on opposite sides of the vortex layer.

It is known that for Rossby waves within the WKB approximation (assuming a smooth variation in the background flow velocity) there is a dynamic invariant in the form of the enstrophy conservation law, regardless of whether the background flow is zonal or non-zonal [5]. The result obtained for the zonal flow is also preserved for the zonal vortex layer when solving the problem in the Miles Ribner formulation. However, if the flow is not zonal, then the results of the WKB approximation and the results for the vortex layer diverge. The Miles - Ribner problem loses its symmetry properties for Hermitian operators, and the analysis becomes extremely laborious.

According to the classical works (for example, [8-10]), in a linear problem when studying the interaction of waves and currents, the first thing to do is to analyze the vortex layer and its eigenmodes. It is known that in the absence of $\beta$-effect, the vortex layer has no neutral eigenmodes, and the two existing modes are unstable, i.e. they are in the complex plane. Since there are no neutral modes, and the vortex layer cannot coexist with stationary wave solutions, this problem is often simply called the Kelvin - Helmholtz instability problem.

The generalization of the vortex layer to the $\beta$-plane was performed in [11], where an equation with radicals was obtained. In order to solve it, just as in the absence of $\beta$-effect, one radical should be transferred to the right side and then both parts should be squared. However, in this case, it is no longer a quadratic equation, as it was in the absence of the $\beta$-parameter, but a cubic one. It has one real and two complex roots, which in fact are generalized Kelvin - Helmholtz modes. It is fundamentally important, according to the authors of the work [11, p. 85-86] that the neutral root is an "artificially acquired" root that arose as a result of squaring the initial dispersion relation for the eigenmode of the vortex layer.

However, later L. D. Talley [12] made a curious mistake, which began to be repeated many times in subsequent works. L. D. Talley writes out a cubic equation (formula (21) in [12, p. 977]), which coincides with the corresponding equation (2.6) from [11, p. 85], and on its basis makes the following erroneous statements: the author calls this cubic equation the dispersion relation, but this is incorrect (since it is the square of the dispersion relation); states that this dispersion relation has three roots: one real (neutral mode) and two complex ones. But at the same time L. D. Talley [12] does not exclude the neutral mode, as should have been done and as done in [11], and later in [13].

Unfortunately, V. M. Kamenkovich and J. Pedlosky [14], like many other authors who later cited this work by L. D. Talley [12], did not notice this fundamental error, which is why all subsequent results of their studies do not look 
entirely correct. Moreover, since the work of L. D. Talley [12], the concepts are confused and used incorrectly. If L. N. Howard and P. G. Drazin [11] considered neutral solutions as radiation and complex modes as instabilities, then L. D. Talley [12] did not call the neutral solutions radiation, but invented the new term "pure radiation" using the term "radiation" for one of the unstable modes. However, in fact, this is just a mode localized in the vicinity of the vortex layer. Unfortunately, this erroneous terminology was not fixed, and many subsequent works by various authors.

There are two qualitatively different approaches to problems with a discontinuous velocity profile. The most famous of them is presented in the monograph [8], in which solutions for the vortex layer are sought immediately in the form of functions strictly damping at infinity. The authors show that if the $\beta$ effect is absent and the density field is constant, then the eigenvalues for the vortex layer mode are strictly complex, i.e. neutral solutions are absent.

However, this raises a reasonable question: how do these results, obtained for absolutely smooth functions of the background flux or in the WKB approximation, can be compared with the results obtained when the background fields are completely smooth. In the linear formulation, both in the WKB approximation and for the vortex layer, the interaction of Rossby waves and zonal flows occurs with the preservation of the adiabatic invariant, i.e. when the enstrophy conservation law is fulfilled. But in fact, for any formulation of such a problem, there is not any interaction, that is, the total flow of enstrophy does not change. In this case, it does not matter whether the wave is refracted smoothly on an alternating zonal flow or a reflected wave is also added.

However, for non-zonal flows the situation is completely different. It is known that zonal unstable solutions become more unstable upon passing to the non-zonal case [14]. Moreover, the statements of all theorems on the stability of zonal flows, considering the upper limiting estimates, for example, the Howard semicircle theorem [15], during the transition to the non-zonal case cease to be true at all [16]. It was shown in [5] that, for smooth analytical functions in the WKB approximation, when the Rossby wave propagates on a non-zonal jet flow in the linear approximation, their interaction occurs within the framework of an adiabatic invariant, which is the enstrophy conservation law. However, with such interaction, the wave actually retains its vorticity and no exchange of vorticity between the wave and the flow occurs. Then the question arises: how stable is the obtained result if we abandon the assumption about the background flow smoothness variation? In this paper, we provide an answer to this question.

The problem of eigenmodes for a zonal vortex layer on the $\beta$-plane was considered in [11, 17], which is mentioned in the monograph [8]. The key point to which attention should be paid is that the authors in their reasoning do not distinguish between the zonal and non-zonal flow. They consider "just a flow", although in reality their equations are derived strictly for the zonal flow.

Looking forward, we are to fix the main line of our reasoning. In our study, we show that for a non-zonal background flow, when this problem is transferred to the $\beta$-plane, the effect of radiation arises, moreover, it is pure radiation (the terminology of L. D. Talley [12]), at which in the refractive and reflection 
coefficients on the real axis appears a pole. Note that this fact was not previously determined. Its simplest explanation: the non-zonal background flow is not actually a solution of the vorticity equation in the $\beta$-plane and, as a consequence, the operator describing linear perturbations in the non-zonal flow is not Hermitian.

The term "pure radiation” is used here because in the literature the simple term "radiation" is used for unstable modes when one of the two unstable modes is simply designated by the author as emitting [12]. However, we do not build the mode in the classical sense of the word, but for the analysis, we use Miles Ribner approach, which was developed in gas dynamics in the 1960s for solving the problem of the interaction of the sound waves with a tangential discontinuity of the velocity and subsequently transferred to the case of a zonal vortex layer for Rossby waves [13, 17, 18].

One of the most common approaches to explaining the formation of vortices and meanders in the ocean is the mechanism of barotropic-baroclinic instability. This mechanism implies that the most unstable mode is first found in the linear problem, then the nonlinear stage is analyzed, and as a result, localized vortex solutions of the "Kelvin's cat's eye” type are obtained [8-10]. With this approach, the following situation is realized: the external force generates only a large-scale flow, but cannot generate wave disturbances.

In our work, we propose an alternative option for the coexistence of largescale flow and wave formations. Its essence is extremely simple: both the stationary shear flow and the wave formations themselves are the results of the action of an external force. That is, an external force not only generates a largescale background current but at the same time is also responsible for the excitation and maintenance of neutral wave formations.

\section{The purpose of the study and problem statement}

The main result obtained by generalizing the vortex layer to the $\beta$-plane consists in the fact that neutral solutions do not appear in this case, i. e. the vortex layer in the strictly zonal case on the $\beta$-plane has no neutral eigensolutions, and the two unstable obtained modes are the legal successors of known varicose and sinusoidal modes. In this paper, we not only generalize the problem of a vortex layer on the $\beta$-plane to the non-zonal case but also show that it is precisely nonzonal that gives the effect of pure radiation.

The main difference of this approach is in a type of boundary conditions at infinity. When constructing a mode, the damping condition at infinity is imposed, at which the solution strictly tends to zero. The formulation of the Miles - Ribner problem requires the solution to be bounded at infinity, and the condition of radiation from a vortex layer is used. In our opinion, this approach is physically more understandable, since when it is used, the vortex layer mode becomes a particular case of this formulation.

Thus, the aim of the study can be briefly formulated as follows: in order to obtain a generalization of the vortex layer problem to the non-zonal case on the $\beta$-plane. 


\section{Main equations and analysis of solutions}

We consider the linearized vorticity equation for the stream function in $\Psi=\Phi(y) \mathrm{e}^{i k(x-c t)}$, where $k$ is the longitudinal component of the wave number; $c$ is a longitudinal component of the phase velocity; $\Phi$ is a stream function, the $x$ axis is directed along with the flow, the $y$-axis is perpendicular to the flow; $t$ is time. In what follows, the longitudinal components $(k, c)$ will be invariants of the problem. The transverse component of the wave number for the incident wave will also be set and assumed to be fixed. The transverse components of the wave vector of the refracted and reflected waves will be found from the dispersion relation taking into account the radiation condition. The group velocities of the refracted and reflected waves are directed from the tangential discontinuity. The amplitude of the incident wave will be considered equal to unity, and all further calculations will be normalized to this value. The amplitudes of the refracted and reflected waves will be found from the matching conditions. The jump in the step of the background flow velocity profile will be considered the variable parameter.

The linear equation of Rossby waves on a shear flow has the form [14]

$$
\Phi_{y y}+\left(\frac{i \beta_{1}}{k(\bar{U}-c)}\right) \Phi_{y}+\left(\frac{\beta_{2}-\bar{U}_{y y}}{\bar{U}-c}-k^{2}-F^{2}\right) \Phi=0
$$

where $\beta_{2}=\beta \cos \theta, \quad \beta_{1}=\beta \sin \theta, \theta$ is an inclination angle of the barotropic planeparallel flow to the parallel; $\bar{U}$ is a flow rate; $F^{2}$ are the eigenvalues of the vertical problem. Then, for the case of constant stratification in the absence of topography, we have the standard expression

$$
F^{2} \equiv \frac{f^{2}(\pi n)^{2}}{N^{2} H^{2}} ; n=0,1,2, \ldots,
$$

where $N$ is Väisälä - Brunt frequency; $f$ is the Earth rotation frequency; $H$ is a depth.

The connection conditions can be derived strictly mathematically, following the formal procedure [8]. The stitching conditions are as follows [14]

$$
\left\{\begin{array}{l}
\Delta\left(\frac{\Phi}{\bar{U}-c}\right)=0, \\
\Delta\left(\Phi_{y}(\bar{U}-c)-\Phi \bar{U}_{y}+\frac{i \beta_{1}}{k} \Phi\right)=0,
\end{array}\right.
$$

where $\Delta$ is a classical jump of values through the vortex layer.

In the Miles - Ribner formulation, we connect three functions: an incident wave of unit amplitude and two waves - a reflected one, with a reflection coefficient $R$, and a transmitted one, with a transmission coefficient $T$. The group velocity of the incident wave is directed towards the step, and the reflected and refracted waves have the group velocity directed away from the step (figure). Since the background flow is considered longitudinally homogeneous and stationary in time, we have two cyclic variables that generate two invariants (integrals of motion) - the longitudinal wavenumber and the wave frequency. 


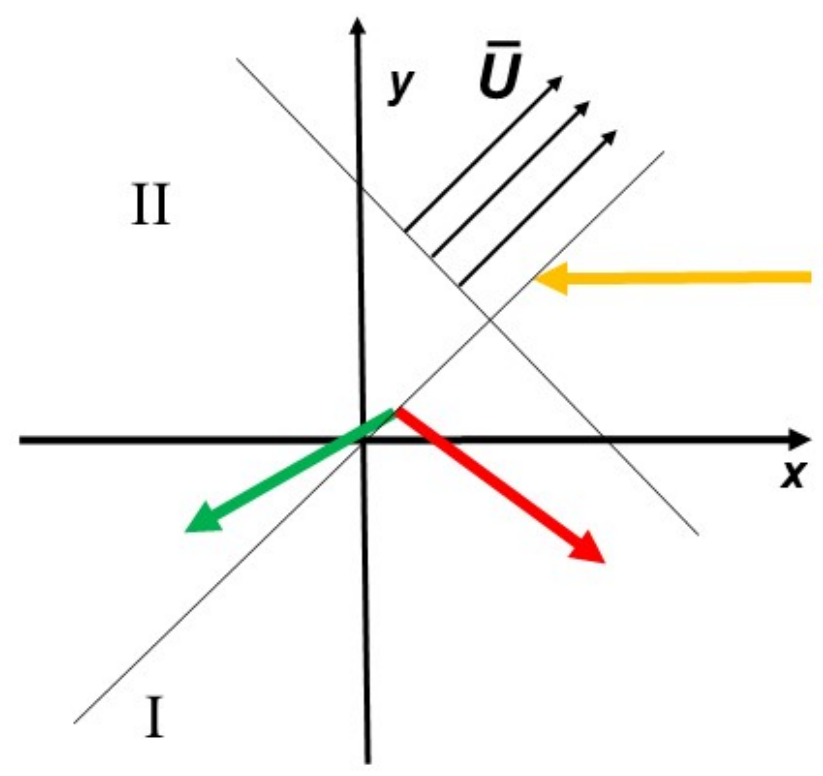

Vortex layer on the non-zonal flow. Incident (yellow arrow), refracted (green arrow), and reflected (red arrow) waves

So, we are looking for a solution (2) in the form

$$
\left\{\begin{array}{l}
\Phi=\exp \left(i l_{1} y\right)+R \exp \left(i l_{0} y\right) \\
\Phi=T \exp \left(i l_{2} y\right)
\end{array}\right.
$$

where $l_{1}, l_{0}, l_{2}$ are transverse components of the wave number of the incident, reflected and refracted waves, respectively. For a self-adjoint operator, in the case of a purely zonal vortex layer, the components of the wavenumber $1_{0}, 1_{1}$ were linked by the relation $l_{0}=-l_{1}$. In our case, the self-adjoint property of the operator is absent, and the symmetry property for Hermitian operators disappears. In this case, we have the following dispersion relation:

$$
\omega=-\frac{\left(\beta_{2} k-\beta_{1} l\right)}{k^{2}+l^{2}+F^{2}}+k \bar{U}
$$

where $\omega$ is a wave frequency, $\omega=k c$.

Further, the dispersion relation (4) will be rewritten in the following form:

$$
l=\frac{\beta_{1}}{2(\omega-k \bar{U})} \pm \sqrt{\left(\frac{\beta_{1}}{2(\omega-k \bar{U})}\right)^{2}-k^{2}-F^{2}-\frac{\beta_{2} k}{(\omega-k \bar{U})}} .
$$


Choosing a sign for the root is determined by the direction of the group velocity in the transverse direction. Then the expressions for the components of the group velocity have the form

- in the transverse direction

- in the longitudinal direction

$$
C_{\text {gr. } y}=\frac{\partial \omega}{\partial l}=\frac{2 \beta_{2} k l-\beta_{1}\left(l^{2}-k^{2}-F^{2}\right)}{\left(k^{2}+l^{2}+F^{2}\right)^{2}} ;
$$

$$
C_{g r . x}=\frac{\partial \omega}{\partial k}=\frac{\beta_{2}\left(k^{2}-l^{2}-F^{2}\right)-2 \beta_{1} k l}{\left(k^{2}+l^{2}+F^{2}\right)^{2}}+\bar{U} .
$$

Substituting expressions (3) into formulas (2), we find the following expressions for the refraction $T$ and reflection $R$ coefficients:

$$
\begin{aligned}
& T=A / C ; \quad R=-B / C ; \\
& A=\left(l_{0}-l_{1}\right)\left(\bar{U}_{1}-c\right)\left(\bar{U}_{2}-c\right) ; \\
& B=\left(\bar{U}_{1}-c\right)^{2} l_{1}-\left(\bar{U}_{2}-c\right)^{2} l_{2}+\frac{\beta_{1}}{k}\left(\bar{U}_{1}-\bar{U}_{2}\right) ; \\
& C=\left(\bar{U}_{1}-c\right)^{2} l_{0}-\left(\bar{U}_{2}-c\right)^{2} l_{2}+\frac{\beta_{1}}{k}\left(\bar{U}_{1}-\bar{U}_{2}\right) .
\end{aligned}
$$

A qualitative analysis of the obtained relations demonstrates the fact that if the jump of the background flow velocity field $\left(\bar{U}_{1}-\bar{U}_{2}\right) \rightarrow 0$, then $R \rightarrow 0, T \rightarrow 1$. Note that the presence of a reflected wave in the solution does not detract from the generality of the approach, even when the step value is zero. The fact is that in this case, the reflection coefficient is zero, and then the reflected wave is automatically excluded.

Analytically verifying the existence of the motion integral, as was done in [13], is rather difficult. However, there are no obstacles for numerical modeling along this path. It is easy to see that the integral is not actually conserved. To see this, it is enough to consider the case when the denominator of the refractive and reflection indices vanishes. Mathematically, this situation is called a pole. The poles of refraction and reflection coefficients are sometimes interpreted as eigenmodes of the vortex layer. In the physical sense, this means that there is a wave of unit amplitude incident on the vortex layer and two waves of infinite amplitude propagating from the vortex layer in different directions. An infinitesimal "seed" wave incident on the vortex layer will cause a disproportionate response in the form of reflected and refracted waves. In particular, one of the solutions may be a wave propagating upstream in the opposite direction (see the Appendix). 
For the classical vortex layer and the vortex layer on the $\beta$-plane in the zonal case, there are no solutions with poles on the real axis. More precisely, formally, of course, the poles exist, but they lie in the complex plane. In contrast, for the nonzonal case, such a solution with a pole on the real axis exists.

In the general case, due to the absence of symmetries in the problem, it is necessary to consider eight variants of the wave incidence on the vortex layer, which is a one-dimensional flow of the "step of infinite length" type. In this work, we will restrict ourselves to analyzing only one case.

Let the vortex layer region be divided into two parts: region I (located in the lower part of the step) and region II (in the upper part of the step). Let the wave fall from the bottom to the top (Figure). We will demonstrate that in this situation a pole on the real axis exists.

Let both velocities be positive, while $U_{2}-U_{1} \equiv 2 \delta_{1}>0$. Then, in dimensionless form, we obtain the equations

$$
\begin{gathered}
c_{1}=\left(c-\frac{U_{1}+U_{2}}{2}\right) / \delta_{1}, \quad \gamma^{2} \equiv k^{2} /\left(k^{2}+F^{2}\right), \quad U_{2}-U_{1} \equiv 2 \delta_{1}, \quad s_{1}=\beta /\left(2\left(k^{2}+F^{2}\right) \delta_{1}\right)(7) \\
\left(c_{1}+1\right)^{2}\left[\frac{s_{1} \sin \theta}{c_{1}+1}+\left(\left[\frac{s_{1} \sin \theta}{c_{1}+1}\right]^{2}-\gamma^{2}-2 \gamma^{2} \frac{s_{1} \cos \theta}{c_{1}+1}\right)^{1 / 2}\right]- \\
-\left(c_{1}-1\right)^{2}\left[\frac{s_{1} \sin \theta}{c_{1}-1}-\left(\left[\frac{s_{1} \sin \theta}{c_{1}-1}\right]^{2}-\gamma^{2}-2 \gamma^{2} \frac{s_{1} \cos \theta}{c_{1}-1}\right)^{1 / 2}\right]-4 s_{1} \sin \theta=0 .
\end{gathered}
$$

The "plus" and "minus" signs in front of the root in different lines of formula (8) correspond to reflected and refracted waves, respectively: plus in the top line gives negative group velocity along the $y$-axis (reflected wave); the minus in the second line gives a positive group velocity (refracted wave). It is easy to see that if the inclination angle of the flow is equal to zero $(\sin \theta=0)$, i. e. the flow is zonal, then squaring equation (8), we obtain exactly the cubic equation (5) from [17, p. 108]: $c_{0}^{3}+1.5 s_{0} c_{0}^{2}+c_{0}+0.5 s_{0}=0$, which is at the same time a generalization of equation (2.6) from [11, p. 85], while $s$ is the dimensionless parameter from [18] and is related to the dimensionless parameter $a$ from [11] by the relation $a=4 s$. The physical meaning of formula (8) is as follows. If the flow is zonal, then, as shown analytically in [17], the interaction of the vortex layer with the waves occurs within the enstrophy conservation law, that is, the enstrophy of the incident wave is equal to the sum of the reflected and refracted waves fluxes. There is a redistribution of enstrophy between three waves: incident, reflected, and refracted, but the total enstrophy does not change.

It is known that for a zonal flow, the consequences of the enstrophy conservation law and the energy conservation law coincide due to the degeneracy of the zonal problem [5]. The refractive and reflection coefficients have no poles on the real axis, the denominator is $C \neq 0$, therefore, no modes corresponding to 
pure radiation [12] are observed, and the poles lie in the complex plane. This means that for a strictly zonal flow different approaches give the same result: 1 ) a continuous profile of the background flow velocity and 2) a piecewise smooth vortex layer, i.e. the enstrophy conservation law as well as the energy conservation law are fulfilled, and the interaction between the flow and wave disturbances are absent.

The enstrophy conservation law is not satisfied for a non-zonal flow for a vortex layer. We demonstrate this with a simple example. For simplicity, we assume that the incident wave moves from below from region I, where the background flow velocity field is zero, to region II. For further analysis, for simplicity of calculations, the following initial parameters were chosen: $\cos \theta=0.6 ; \sin \theta=0.8 ; \quad F^{2}=1 ; k=0.5 ; \beta=10 ; \omega=1$. From the dispersion relation (6) we obtain the transverse components of the wave number: $l_{1}=4-\sqrt{11.75} \approx 0.57 ; \quad l_{0}=4+\sqrt{11.75} \approx 7.43$. In accordance with the radiation condition, we choose a minus in front of the root in expression (6) for the transverse component of the wave number of the refracted wave. Then at $\bar{U}=0$ we get the following set of parameters:

$$
\begin{gathered}
l_{2}=4-\sqrt{11.75}=0.5721 ; \quad C_{g r . y 1}=4.346 ; \quad C_{g r . y 2}=4.346 ; \\
C_{g r . y 0}=-0.1215 ; \quad T r=1 ; \quad \operatorname{Ref}=0 ; \quad C=7.42 .
\end{gathered}
$$

A simple check confirms that the incident and reflected waves have the values of group velocities along the transverse coordinate, which are opposite in sign.

With a gradual increase in the step to $\bar{U}=1.856$ value, the denominator $C$ remains positive, $C \rightarrow 0$. We find $l_{2}=0.3876 ; C_{\text {gr. } y 2}=5.673 ; C=0.0072$; $\operatorname{Tr}=272 ; \quad \operatorname{Ref}=3770$. Note that the reflection and transmission coefficients have already increased by three orders of magnitude (!).

At a value $\bar{U}=1.857$, the denominator of $C$ is already negative: $C<0$. In this case $l_{2}=0.3875 ; \quad C_{g r . y 2}=5.674027 ; \quad C=-0.0082 ; \quad \operatorname{Tr}=-228 ; \quad \operatorname{Ref}=-3184$. The effect appears before the critical layer arises in the problem, from which it follows that the waves under consideration are not the waves of negative energy. At the selected parameters $(\omega=1$, longitudinal component $k=0.5)$, the phase velocity in the longitudinal direction is 2 .

We carry out the calculation for the same parameters using the dimensionless equation (8): we find $\gamma^{2}=0.2$ at $\bar{U}=1.856$. Then we get $s=4.3103$, and the left side of (8) takes a positive value of 0.00373 . At $\bar{U}=1.857$ we get $s=4.3080$, and the expression takes on a negative value $(-0.01148)$. Therefore, the root of the equation is between these values and, most importantly, it exists.

If the parameter $\beta$ is reduced, then in the calculation it turns out that the pole appears at a smaller value of the step. The calculation gives the following figures: $\beta=10$ at $\bar{U}=1.857 ; \beta=9$ at $\bar{U}=1.8331 ; \beta=7$ at $\bar{U}=1.7546$. 
If we take the barotropic case $F^{2}=0$, then the pole still exists, but already for smaller values of the velocity jump.

If the wave falls not from the south, but the north, then with the same parameters but for an inverted step there is no pole, the denominator does not vanish, which again demonstrates the absence of symmetry properties in the problem, which, as it is easy to see, is due to the last, non-zonal term in equation (7). The critical value of the step size is much larger and lies behind the critical layer:

$$
\begin{gathered}
\bar{U}=-2.935 ; \quad s=0.6 ; \quad p=1 ; \quad k=0.5 ; \quad b=10 ; \quad \omega=1 ; \\
C=-0.0089935 ; \quad \operatorname{Tr}=1881 ; \quad \operatorname{Ref}=761 ; \\
C_{g r . y 1}=-0.0121505 ; \quad C_{g r . y 0}=0.4346225 ; \quad C_{g r . y 2}=-0.0371691
\end{gathered}
$$

We note the following circumstance. For the non-zonal case, these results are a consequence of the fact that expression (1) is not a Hermitian operator, in contrast to the zonal case. Perhaps this fact is the key to understanding the results obtained in this problem.

\section{Conclusion}

We have demonstrated that for a non-zonal flow interacting with Rossby waves, there is a new class of solutions that can be interpreted as pure emission of Rossby waves by a non-zonal flow. This approach will eliminate the resulting confusion in terminology when instabilities are called radiation, and radiation pure radiation, while the authors, using the term "radiation", actually analyze the instabilities.

In other words, the novelty of our work is as follows. When analyzing the nonzonal case of a linear problem, many authors came, in fact, to the same conclusion: unstable solutions become more unstable and all theorems and constraints on instabilities for the zonal case cease to be true in the non-zonal case [17]. We have demonstrated that in the non-zonal case there is a completely new class of solutions that is absent in the zonal case. We have also shown that it is precisely the nonzoning that gives the effect of pure radiation. This class of solutions corresponds to the classical definition of radiation, in contrast to unstable solutions, incorrectly called radiation.

\section{Appendix \\ On non-zonality of vortex layer}

The vorticity equation on the $\beta$-plane in the coordinate system rotated by an angle $\theta$ to the parallel has the form

$$
\frac{d_{h}}{d t}\left[\nabla_{h}^{2} \Psi+\left(\frac{1}{S} \Psi_{z}\right)_{z}+\beta(y \cos \theta+x \sin \theta)\right]=0 .
$$

We will look for solutions in the form 


$$
\Psi(x, y, z, \theta, t)=\Psi_{0}(y, z, \theta) .
$$

Substituting expression (A.2) into formula (A.1), we obtain

$$
\frac{\partial \Psi_{0}(y, z, \theta)}{\partial y} \beta \sin \theta=0
$$

It follows from the equation (A.3):

1) if the angle $\theta$ is equal to zero (the $x$-axis is directed along the parallel - the case of zonal flow), equation (A.3) is fulfilled for any function $\Psi_{0}(y, z, \theta)$. Consequently, any plane-parallel stationary flow is a solution of the nonlinear vorticity equation on the $\beta$-plane. The result is well known [8];

2 ) if the angle $\theta$ is not equal to zero (the $x$-axis is not directed along the parallel - the case of a non-zonal flow), then there are no nontrivial solutions.

Consequently, for the existence of a non-zonal plane-parallel stationary flow within the framework of the nonlinear vorticity equation on the $\beta$-plane, the presence of an external force is required, i.e. we must include some external force in the right-hand side of equation (A.3). Consequently, in the original linear equation in the non-zonal case (1), the dependence $\bar{U}=\bar{U}(F)$ is implicitly assumed. This means that from equation (1) it follows that linear wave solutions depend on an external force, that is, not only the non-zonal flow itself but also the wave solutions for the non-zonal case are the result of the direct action of an external factor.

Thus, if the vortex layer is purely zonal, and the linear operator is self-adjoint, then small perturbations depend on the profile of the large-scale flow and are unstable. If the vortex layer is not zonal, then the linear operator is not self-adjoint, and then the external force determines the shear stationary flow and wave disturbances.

The influence of an external force for the non-zonal case is not limited only to the action of a large-scale flow. When waves interact with a large-scale stationary flow, upon reaching a certain threshold value, a new class of solutions appears, which can be interpreted as flow radiation, or as a direct generation of wave disturbances by an external force. This result explains how a non-zonal jet flow and wave formations moving in the opposite direction can exist simultaneously.

This problem, even in a simple formulation, requires further analysis. In particular, it is necessary to consider physically more realistic velocity profiles e. g. like "hat" (rectangle).

\section{REFERENCES}

1. Gnevyshev, V.G. and Shrira, V.I., 1989. Dynamics of Rossby-Wave Packets in the Vicinity of Zonal Critical Layers with Account of Viscosity. Izvestiya Akademii Nauk SSSR, Fizika Atmosfery i Okeana, 25(10), pp. 1064-1074 (in Russian). 
2. Gnevyshev, V.G., and Shrira, V.I., 1989. Kinematics of Rossby Waves on Non-Uniform Meridional Current. Okeanologiya, 29(4), pp. 543-548 (in Russian).

3. Gnevyshev, V.G. and Shrira, V.I., 1989. Estimates for Parameters of Barotropic-Baroclinic Instability of Zonal Flows on a $\beta$-Plane. Doklady Mathematics, 34(5), pp. 418-420.

4. Gnevyshev, V.G., and Shrira, V.I., 1989. Monochromatic Rossby-Transformation in Zonal Flow Critical Layers. Izvestiya Akademii Nauk SSSR, Fizika Atmosfery i Okeana, 25(8), pp. 852-862 (in Russian).

5. Gnevyshev, V.G., Badulin, S.I. and Belonenko, T.V., 2020. Rossby Waves on Non-Zonal Currents: Structural Stability of Critical Layer Effects. Pure and Applied Geophysics, 177, pp. 5585-5598. https://doi.org/10.1007/s00024-020-02567-0

6. Gnevyshev, V.G., Frolova, A., Kubryakov, A.A. and Sobko, Yu.V., 2019. Interaction between Rossby Waves and a Jet Flow: Basic Equations and Verification for the Antarctic Circumpolar Current. Izvestiya, Atmospheric and Oceanic Physics, 55(5), pp. 412-422. doi:10.1134/S0001433819050074

7. Gnevyshev, V.G. and Belonenko, T.V., 2020. The Rossby Paradox and Its Solution. Gidrometeorologiya i Ekologiya. Hydrometeorology and Ecology (Proceedings of the Russian State Hydrometeorological University), 61, pp. 480-493. doi:10.33933/20742762-2020-61-480-493 (in Russian).

8. LeBlond, P., and Mysak, L., 1978. Waves in the Ocean. Amsterdam: Elsevier Scientific Publishing Company, 602 p.

9. Pedlosky, J., 1987. Geophysical Fluid Dynamics. New York: Springer, 710 p. https://doi.org/10.1007/978-1-4612-4650-3

10. Flierl, G.R., Malanotte-Rizzoli, P. and Zabusky, N.J., 1987. Nonlinear Waves and Coherent Vortex Structures in Barotropic $\beta$-plane Jets. Journal of Physical Oceanography, 17(9), pp. 1408-1438. https://doi.org/10.1175/1520-0485(1987)017<1408:NWACVS>2.0.CO;2

11. Howard, L.N. and Drazin, P.G., 1964. On Instability of Parallel Flow of Inviscid Fluid in a Rotating System with Variable Coriolis Parameter. Journal of Mathematics and Physics, 43(1-4), pp. 83-99. https://doi.org/10.1002/sapm196443183

12. Talley, L.D., 1983. Radiating Barotropic Instability. Journal of Physical Oceanography, 13(6), pp. 972-987. https://doi.org/10.1175/1520-0485(1983)013<0972:RBI>2.0.CO;2

13. Stepanyants, Yu.A. and Fabrikant, A.L., 1989. Propagation of Waves in Hydrodynamic Shear $\begin{array}{llll}\text { Flows. Soviet } \quad \text { Physics } & \text { 3spekhi, }\end{array}$ doi:10.1070/PU1989v032n09ABEH002757

14. Kamenkovich, I.V. and Pedlosky, J., 1996. Radiating Instability of Nonzonal Ocean Currents. Journal of Physical Oceanography, 26(4), pp. 622-643. https://doi.org/10.1175/15200485(1996)026<0622:RIONOC>2.0.CO;2

15. Drazin, P.G. and Howard, L.N., 1966. Hydrodynamic Stability of Parallel Flow of Inviscid Fluid. In: G. G. Chernyi, H. L. Dryden, P. Germain, L. Howarth, W. Olszak, W. Prager, R. F. Probstein and H. Ziegler, Eds., 1966. Advances in Applied Mechanics. New York: Academic Press. Vol. 9, pp. 1-89. https://doi.org/10.1016/S0065-2156(08)70006-1

16. Kobayashi, S. and Sakai, S., 1993. Barotropic Unstable Modes in Zonal and Meridional Channel on the Beta-Plane. Geophysical \& Astrophysical Fluid Dynamics, 71(1-4), pp. 73103. doi:10.1080/03091929308203598

17. Fabrikant, A.L., 1987. The Reflection of Rossby Waves from the Surface of a Tangential Velocity Discontinuity. Izvestiya, Atmospheric and Oceanic Physics, 23(1), pp. 79-81.

18. Gnevyshev, V.G. and Shrira, V.I., 1990. On the Evaluation of Barotropic-Baroclinic Instability Parameters of Zonal Flows on a Beta-Plane. Journal of Fluid Mechanics, 221, pp. 161-181. https://doi.org/10.1017/S0022112090003524

About the authors:

Vladimir G. Gnevyshev, Senior Research Associate, Shirshov Institute of Oceanology of Russian Academy of Sciences (5 Nakhimovsky pr., Moscow, 117997, Russian Federation), Ph. D. (Phys.-Math.), 
ORCID ID: 0000-0001-6654-5570, ResearcherID: AAZ-6352-2021, Scopus Author ID: 6507346231, avi9783608@gmail.com

Tatyana V. Belonenko, Professor, Oceanology Department, Saint Petersburg State University (7-9 Universitetskaya emb., St. Petersburg, 199034, Russian Federation), Dr. Sci. (Geogr.), ResearcherID: K-2162-2013, Scopus Author ID: 6507005889, btvlisab@yandex.ru

Contribution of the co-authors:

Vladimir G. Gnevyshev - the main idea, formulation of the problem statement, analysis of the data, writing and editing the text of the paper

Tatyana V. Belonenko - the main text of the paper, formulation of purposes and objectives of the study, analysis of literature, graphic material

All the authors have read and approved the final manuscript.

The authors declare that they have no conflict of interest. 